\title{
SUPLEMENTAÇÃO DIETÉTICA DE SELÊNIO PARA FRANGOS DE CORTE E SEUS EFEITOS SOBRE O EMPENAMENTO
}

\author{
(Selenium dietary supplementation of broilers and its effects on feathering)
}

DAHLKE, F. ${ }^{1}$; GONZALES, E. ${ }^{2}$; FURLAN. R.L. ${ }^{3}$ GADELHA, A. C. ${ }^{4}$; MAIORKA, A. ${ }^{1}$; ALMEIDA, J.G. ${ }^{5}$

\author{
${ }^{1}$ Departamento de Zootecnia, UFPR, Curitiba, PR; \\ ${ }^{2}$ Faculdade de Medicina Veterinária e Zootecnia, Unesp/Botucatu, SP; \\ ${ }^{3}$ Faculdade de Ciências Agrárias e Veterinárias, Unesp, Jaboticabal, SP; \\ ${ }^{4}$ Departamento de Zootecnia, Universidade Federal do Ceará, Fortaleza, CE; \\ ${ }^{5}$ Departamento de Zootecnia, Universidade Federal do Rio Grande do Sul, RS.
}

RESUMO - Foi avaliado o empenamento de frangos de corte alimentados com dietas suplementadas com diferentes fontes e níveis de selênio (Se), criados sob diferentes condições de temperatura. Foram utilizados 720 pintainhos, Cobb-500, em um delineamento inteiramente ao acaso em esquema fatorial $3 \times 2 \times 2$ (3 temperaturas ambiente: termoneutra, fria e quente; 2 níveis de suplementação de Se: nível A e B; e 2 fontes de Se: orgânica e inorgânica), com 4 repetições de 15 aves. Os níveis A e B de Se na ração inicial (1 a 21 dias) e de crescimento (22 a 42 dias) foram 0,15 e 0,30 mg/kg e 0,20 e 0,40 mg/kg, respectivamente, sendo Selenito de sódio a fonte inorgânica e selenometionina a forma de suplementação orgânica. Os frangos criados no calor apresentaram menor cobertura e crescimento de penas. Aos 21 dias de idade o Se orgânico induziu a um mais rápido empenamento nas regiões pterígeas avaliadas, independente da variação dos níveis de Se estudados. Entretanto, a avaliação feita aos 42 dias mostrou que a suplementação de Se, nas suas diferentes formas e níveis, não interferiu no empenamento nesta idade. Os resultados são indicativos de que a suplementação de Se na sua forma orgânica parece ser a melhor maneira de se obter um bom empenamento dos frangos de corte em uma fase crítica para a sua homeostase térmica, isto é, até os 21 dias de idade.

Palavras-chave: empenamento, frangos de corte, selênio, temperatura ambiente.

ABSTRACT - This study evaluated the feathering of broiler fed supplemented diet with different selenium (Se) sources and levels, raised under different environmental temperatures. Seven hundred twenty broiler chicks were raised in a complete randomized design with $3 \times 2 \times 2$ factorial schedule ( 3 environmental temperatures: cold, neutral and hot; two levels and two Se sources) with 4 repetitions of 15 birds. The levels of Se in rations for initial and growth phases were: 0.15 and $0.30 \mathrm{mg} / \mathrm{kg}$; and 0.20 and $0.40 \mathrm{mg} / \mathrm{kg}$, respectively. The organic and inorganic sources of selenium were selenomethionine and sodium selenite, respectively. There was a feathering reducing of the broilers housed in the hot temperature. At 21 days of birth organic selenium induces a fast feathering growth on the pteriloses evaluated regions. However, at 42 days, different selenium levels or sources did not affect the broiler feathering. These data show that organic selenium supplementation is the better way to get a good broiler feathering on critical phase of thermal homeostasis, until 21 days of birth.

Key-words: broiler, feathering, environmental temperature, selenium. 


\section{Introdução}

Embora a redução do empenamento possa facilitar a dissipação de calor em frangos criados em ambiente quente, uma boa cobertura de é necessária para a manutenção da integridade da carcaça. Diversas lesões verificadas em frangos de corte, como calos de peito, arranhões, lacerações, cortes e outros traumas de pele causados por uma má cobertura de penas resultam em condenações de carcaça nas linhas de abate (MENDES, 2001) Alterações no empenamento são freqüentemente observadas em aves alimentadas com dietas deficientes em Se (JENSEN, et al., 1986). Neste sentido, algumas hipóteses podem ser consideradas. 1. O Se atua no empenamento através da ação do hormônio triiodotironina $\left(T_{3}\right)$, pois é conhecida a participação do Se como modulador enzimático na ativação deste hormônio, que, por sua vez, interfere na reação em cadeia da síntese protéica (BECKETT et al., 1992, HAYASHI, 1993). 2. Devido à sua similaridade com o enxofre, o Se poderia substituir os aminoácidos sulfurados (metionina - Met e cisteína - Cys), na rota metabólica de síntese de queratina, principal componente protéico da pena.

Sabe-se, também, que o Se está envolvido na síntese de aminoácidos contendo enxofre, principalmente na eficiência da transsulfuração, que resulta na formação da Cys a partir da Met (HALPIN e BAKER, 1984). Sendo os aminoácidos sulfurados importantes para o crescimento da pena, principalmente a Cys, uma vez que cerca de $25 \%$ da Cys da dieta é utilizada na síntese da queratina (LEESON e SUMMERS, 1997), supõe-se que alterações desta atividade possam afetar a qualidade do empenamento.

Além da conhecida influência sobre o desempenho, parece que o empenamento também é alterado pela temperatura ambiente. COOPER e WASHBURN (1998) e WYLIE et al. (2001) verificaram redução da cobertura de penas quando as aves foram expostas ao calor. Contraditoriamente, YALCIN et al. (1997) constataram uma melhora no empenamento em alta temperatura, e EDENS et al. (2000), por sua vez, observaram maior crescimento da pena em ambiente frio.

Da mesma forma, os hormônios $T_{3}$ e $T_{4}$ podem ser alterados pela temperatura ambiente. Exposição da ave a temperaturas baixas promove aumento dos níveis sangüíneos de $\mathrm{T}_{3}$, com efeito inverso em temperaturas elevadas (HADDAD e MASHALY, 1989). Altos níveis destes hormônios promovem maiores consumos de oxigênio, ativam a síntese e metabolismo de proteínas, carboidratos e lipídios (LAWRENCE e FOWLER, 1997) e, supõe-se, que aumentam a atividade metabólica das papilas formadoras de pena (LUCAS e STETTENHEIM, 1972). Como existe um aumento na atividade da 5 'deiodinase, enzima ativadora do hormônio $T_{3}$, como conseqüência do aumento da ingestão de Se (JIANHUA et al. ,2000), acredita-se que alterações do empenamento causadas pela ação da temperatura ambiente possam ser corrigidas com níveis dietéticos mais altos desse micromineral.

Desta forma, o presente estudo objetivou avaliar o efeito da suplementação dietética de selênio sobre o empenamento em frangos de corte submetidos a estresse por calor e frio.

\section{Material e Métodos}

Foram utilizados 720 frangos de corte, machos, Cobb-500 ${ }^{\circledR}$ criados de 1 a 42 dias de idade, em câmaras climáticas, sob três condições de temperatura ( $\mathrm{T}$ ): Temperatura Termoneutra (TN) de acordo com a idade das aves ( 5 a 7 dias de idade: $32^{\circ} \mathrm{C}$; 8 a 14 dias de idade: $30^{\circ} \mathrm{C}$; 15 a 21 dias de idade: $26^{\circ} \mathrm{C}$ e de 22 a 42 dias de idade: $24^{\circ} \mathrm{C}$ ); Temperatura Quente (TQ), com a temperatura variando de 3 a $9^{\circ} \mathrm{C}$ acima da temperatura neutra e Temperatura Fria (TF) com variações de 7 a $9^{\circ} \mathrm{C}$ abaixo da temperatura termoneutra.

As aves foram distribuídas nos boxes segundo delineamento inteiramente casualizado, em modelo fatorial $2 \times 2 \times 3$ (níveis de selênio, fontes de selênio e temperaturas ambiente) com 4 repetições de 15 aves cada.

As rações, a base de milho e farelo de soja, foram isonutritivas (Tabela 1 ), e para a obtenção dos níveis desejados de selênio, foram suplementados à dieta basal $0,15 \mathrm{mg}$ e $0,3 \mathrm{mg}$ 
Suplementação dietética de selênio para frangos de corte e seus efeitos sobre o empenamento...

de Se por kg de ração, na fase inicial; 0,2 mg e $0,4 \mathrm{mg}$ de Se/kg de ração na fase de crescimento. O Se foi adicionado à dieta na forma inorgânica (selenito de sódio $-\mathrm{Na}_{2} \mathrm{SeO}_{3}$ ) ou orgânica (Selenometionina - SO). As dietas foram assim constituídas: Ração1 (R1) - 0,15 $\mathrm{mg}$ de Se fornecido através de selenito de sódio, Ração 2 (R2) - 0,30 mg de Se fornecido através de selenito de sódio, Ração 3 (R3) - 0,15 mg de Se fornecido através de Se orgânico e Ração 4 (R4) - 0,30 mg de Se fornecido através de Se orgânico, para a fase inicial. Para a fase de crescimento as dietas foram: Ração 5 (R5) - 0,20 $\mathrm{mg}$ de Se fornecido através de selenito de sódio, Ração 6 (R6) - 0,40 mg de Se fornecido através de selenito de sódio, Ração 7 (R7) - 0,20 mg de Se fornecido na forma de Se orgânico e Ração 8 (R8) - 0,40 mg de Se fornecido na forma de Se orgânico (TABELA 1).

Aos 21 e 42 dias de idade foram selecionadas 8 aves por tratamento, representativas do peso médio da unidade experimental para o estudo do padrão de empenamento, através de duas metodologias. A primeira metodologia consistiu em avaliar visualmente as aves, dando-lhes um escore de 0 a 5 , de acordo com a densidade de empenamento e estágio de desenvolvimento das penas nas regiões: dorso (dorsopelvico), peito (pectoral + esternal) , pernas (femural), abdômen (Medial e Lateral) e asa (umeral e cobertura marginal superior do prepatagium), seguindo o critério adotado por EDENS et al. (2001).

A segunda metodologia, comprimento das penas (WYLIE et al., 2001), utilizou também 8 aves por tratamento aos 28 e 43 dias de idade. Para esta análise, a região dorso pélvica (dorso) foi dividida em duas partes, cranial e caudal. Na porção central da região caudal do trato foi medido aleatoriamente o comprimento de 5 penas por ave e a média utilizada para análise estatística. Da mesma forma foi medido o comprimento de 5 penas da porção proximal da região femoral, das aves selecionadas. Ainda, foi medido o comprimento da segunda rêmige primária, da asa esquerda.

Os dados obtidos foram submetidos à análise de variância através do procedimento General Linear Model (GLM) do programa SAS ${ }^{\circledR}$ (SAS Institute, 2002) e em caso de diferença estatística significativa as médias foram comparadas pelo teste de Tukey à $5 \%$ de probabilidade.

\section{Resultados}

Aos 21 dias, os frangos de corte criados em ambiente quente apresentaram uma menor cobertura de penas que os frangos criados em temperatura fria ou em condição de termoneutralidade (TABELA 2). Os dados observados nesse experimento mostraram, também, que o selênio orgânico induziu um mais rápido empenamento total da ave aos 21 dias de idade. A variação nos níveis de $\mathrm{Se}$, no entanto, não alterou a cobertura de penas.

A redução do empenamento, verificada em aves criadas em condição de calor permaneceu até os 42 dias de idade (TABELA 3 ). O crescimento desta estrutura não sofreu alteração em função do aumento do nível dietético de Se ou pela variação das formas com que esse micromineral foi fornecido aos frangos.

A redução do empenamento em altas temperaturas verificada através do método subjetivo de avaliação (escore) foi confirmada quando medido o comprimento das penas do dorso e perna das aves aos 35 e 42 dias (TABELA 4). Também nestas idades, confirmando os achados do primeiro método, não houve influência da fonte de selênio ou dos níveis empregados.

O crescimento das remiges não sofreu influência da temperatura, níveis ou fonte de selênio, aos 35 e 42 dias de idade.

\section{Discussão}

O menor empenamento, verificado em todo o período experimental, das aves criadas em condição de estresse térmico por calor, demonstra a priorização na manutenção da homeostase em detrimento ao crescimento desta estrutura tegumentar. Estes dados corroboram COOPER e WASHBURN (1998) ao verificarem uma redução no empenamento quando as aves foram expostas a temperatura de $32^{\circ} \mathrm{C}$ até os 49 dias de idade. Relatos de GERAERT et al. (1996) dão conta de que a redução do empenamento acontece somente 
quando a ave é exposta ao calor por mais de duas semanas. De acordo com WYLIE et al. (2001), a redução do empenamento pode ser uma resposta adaptativa para a melhor dissipação de calor em climas quentes. GERAERT et al. (1996) observaram menor empenamento nas aves criadas no calor do que nas demais temperaturas, utilizando programa de alimentação pair feeding. Estes achados indicam a interferência de outros fatores, além da redução da ingestão, no empenamento.

TABELA 1 - COMPOSIÇÃO (\%) DAS DIETAS EXPERIMENTAIS NA FASE INICIAL (1- 21DIAS DE IDADE) E FASE DE CRESCIMENTO (22-42 DIAS DE IDADE).

\begin{tabular}{|c|c|c|c|c|c|c|c|c|}
\hline & \multicolumn{4}{|c|}{ 1-21 dias } & \multicolumn{4}{|c|}{$22-42$ dias } \\
\hline Ingredientes (\%) & $\mathrm{R}^{3}{ }^{3}$ & R2 & R3 & R4 & R5 & R6 & R7 & R8 \\
\hline Milho & 51,00 & 51,00 & 51,00 & 51,00 & 56,60 & 56,60 & 56,60 & 56,60 \\
\hline Farelo de soja & 40,43 & 40,43 & 40,43 & 40,43 & 34,68 & 34,68 & 34,68 & 34,68 \\
\hline Óleo de soja & 4,59 & 4,59 & 4,59 & 4,59 & 5,10 & 5,10 & 5,10 & 5,10 \\
\hline Fosfato bicálcico & 1,74 & 1,74 & 1,74 & 1,74 & 1,72 & 1,72 & 1,72 & 1,72 \\
\hline Calcário & 1,40 & 1,40 & 1,40 & 1,40 & 1,22 & 1,22 & 1,22 & 1,22 \\
\hline Sal comum & 0,40 & 0,40 & 0,40 & 0,40 & 0,32 & 0,32 & 0,32 & 0,32 \\
\hline Suplemento vit. /mineral ${ }^{2}$ & 0,10 & 0,10 & 0,10 & 0,10 & 0,10 & 0,10 & 0,10 & 0,10 \\
\hline DL-metionina & 0,29 & 0,29 & 0,29 & 0,29 & 0,21 & 0,21 & 0,21 & 0,21 \\
\hline Butil Hidroxitolueno & 0,03 & 0,03 & 0,03 & 0,03 & 0,03 & 0,03 & 0,03 & 0,03 \\
\hline Porção variável ${ }^{1}$ & 0,02 & 0,02 & 0,02 & 0,02 & 0,02 & 0,02 & 0,02 & 0,02 \\
\hline Composição calculada & \multicolumn{4}{|c|}{$1-21$ dias } & \multicolumn{4}{|c|}{$22-42$ dias } \\
\hline Energia Metabolizável (kcal/kg) & \multicolumn{4}{|c|}{3050} & \multicolumn{4}{|c|}{3150} \\
\hline Proteína Bruta (\%) & \multicolumn{4}{|c|}{22,00} & \multicolumn{4}{|c|}{20,00} \\
\hline Cálcio (\%) & \multicolumn{4}{|c|}{1,00} & \multicolumn{4}{|c|}{0,90} \\
\hline Fósforo Disponível (\%) & \multicolumn{4}{|c|}{0,44} & \multicolumn{4}{|c|}{0,43} \\
\hline Sódio (\%) & \multicolumn{4}{|c|}{0,20} & \multicolumn{4}{|c|}{0,15} \\
\hline Metionina (\%) & \multicolumn{4}{|c|}{0,57} & \multicolumn{4}{|c|}{0,45} \\
\hline Metionina + Cistina (\%) & \multicolumn{4}{|c|}{0,95} & \multicolumn{4}{|c|}{0,82} \\
\hline Lisina (\%) & \multicolumn{4}{|c|}{1,20} & \multicolumn{4}{|c|}{1,00} \\
\hline Potássio (\%) & \multicolumn{4}{|c|}{0,90} & \multicolumn{4}{|c|}{0,83} \\
\hline Cloro (\%) & \multicolumn{4}{|c|}{35} & & & \\
\hline
\end{tabular}

1. A porção variável foi constituída de selenit o de sódio (selênio inorgânico) e selenometionina (selênio orgânico) e inerte, a fim de se obter os níveis de 0,15 e $0,30 \mathrm{mg}$ de Se/kg de ração de 1 a 21 dias ; 0,20 $\mathrm{mg}$ e $0,40 \mathrm{mg} / \mathrm{kg}$ de ração de 21 a 42 dias de idade.

2. Suplemento Mineral e Vitamínico: Cada quilograma do produto contém: vitamina A $90.000 U$; vitamina $E$ $20 \mathrm{mg}$; vitamina K 2,5 mg; vitaminaB1 2,0 mg; vitamina B2 6,6 mg; vitamina B12 20,0 mcg; ácido fólico 0,1 mg; ácido pantotênico $10,0 \mathrm{~m}$; niacina $100,0 \mathrm{mg}$, biotina $0,15 \mathrm{mg}$; colina $1,54 \mathrm{~g}$; antioxidante $0,125 \mathrm{mg}$; iodo 0,35 mg; cobre 10,0 mg; ferro 50,0 mg; manganês 88,0 mg; zinco 0,1 mg; enxofre 0,72g; Bacitracina de Zinco 0,03 ; Coxixtac 0,05

\section{Constituições das rações}

Ração 1 (R1) - 0,15 mg de Se fornecido através de selenito de sódio,

Ração 2 (R2) - 0,30 mg de Se fornecido através de selenito de sódio,

Ração 3 (R3) - 0,15 mg de Se fornecido através de Se orgânico,

Ração 4 (R4) - 0,30 mg de Se fornecido através de Se orgânico, para a fase inicial.

Ração 5 (R5) - 0,20 mg de Se fornecido através de selenito de sódio,

Ração 6 (R6) - 0,40 mg de Se fornecido através de selenito de sódio,

Ração 7 (R7) - 0,20 mg de Se fornecido na forma de Se orgânico

Ração 8 (R8) - 0,40 mg de Se fornecido na forma de Se orgânico, para a fase de crescimento 
Suplementação dietética de selênio para frangos de corte e seus efeitos sobre o empenamento...

O melhor empenamento aos 21 dias de idade, verificado nas aves alimentadas com dietas contendo Se orgânico, corroboram os achados de EDENS et al. (2000) e EDENS et al. (2001), que verificaram maior empenamento nas aves que foram alimentadas com dietas adicionadas de Se orgânico na sua formulação. Sendo o selênio orgânico retido nos tecidos com maior eficiência do que o selenito de sódio, (PATON et al., 2002) sendo possível que no período em que exista maior demanda para a síntese de pena, os selenoaminoácidos selenometionina (forma com que o selênio orgânico é apresentado) e selenocisteína sejam mais facilmente usados na síntese de queratina nas penas, poupando os depósitos hepáticos e musculares de cisteína (EDENS et al., 2000).

O crescimento das remiges primárias não sofreu influência da temperatura ou suplementação de Se. Anteriormente ÖZKAN et al. (2002) haviam feito esta observação, verificando que independente do genótipo, a temperatura ambiente ou mesmo o programa de restrição alimentar, não alteraram o crescimento das penas.

TABELA 2 - EFEITO DOS NÍVEIS E FONTES DE SELÊNIO E DA TEMPERATURA AMBIENTE NO EMPENAMENTO DE FRANGOS DE CORTE AOS 21 DIAS DE IDADE.

\begin{tabular}{lcccccc}
\hline Temperatura ambiente & Perna & Abdômen & Dorso & Asa & Peito & Total \\
\hline Quente & $1,87 \mathrm{c}$ & $1,70 \mathrm{c}$ & $1,70 \mathrm{c}$ & $1,96 \mathrm{~b}$ & $1,22 \mathrm{~b}$ & $1,55 \mathrm{c}$ \\
Termoneutra & $2,26 \mathrm{a}$ & $2,11 \mathrm{a}$ & $2,34 \mathrm{a}$ & $2,31 \mathrm{a}$ & $1,62 \mathrm{a}$ & $2,05 \mathrm{a}$ \\
Fria & $2,03 \mathrm{~b}$ & $1,93 \mathrm{~b}$ & $2,08 \mathrm{~b}$ & $2,15 \mathrm{a}$ & $1,38 \mathrm{~b}$ & $1,84 \mathrm{~b}$ \\
\hline \multicolumn{1}{c}{ Fonte de Selênio } & & & & & & \\
\hline Inorgânico & 1,99 & 1,96 & 1,98 & 1,90 & 1,32 & 1,76 \\
Orgânico & 2,12 & 1,86 & 2,12 & 2,08 & 1,48 & 1,87 \\
$\quad$ Nivel de Selênio & & & & & & \\
Nivel "A" & 2,03 & 1,86 & 2,00 & 1,99 & 1,44 & 1,79 \\
Nivel "B" & 2,07 & 1,96 & 2,08 & 2,05 & 1,36 & 1,84 \\
& & & & & & \\
\hline
\end{tabular}

Médias seguidas de letras distintas, dentro de cada fator, diferem entre si pelo teste de Tukey (5\%). *Nível A: 0,15 mg de Se/kg de ração (1 - 21 dias de idade) e 0,20 mg (21- 42 dias de idade) Nível B: 0,30 mg de Se/kg de ração (1-21 dias de idade) e 0,40 mg (21- 42 dias de idade)

TABELA 3 - EFEITO DOS NÍVEIS E FONTES DE SELÊNIO E DA TEMPERATURA AMBIENTE NO EMPENAMENTO DE FRANGOS DE CORTE AOS 42 DIAS DE IDADE.

\begin{tabular}{lllllll}
\hline Temperatura ambiente & Perna & Abdômen & Dorso & Asa & Peito & Total \\
Quente & $3,61 \mathrm{~b}$ & $3,79 \mathrm{~b}$ & $4,53 \mathrm{a}$ & $4,66 \mathrm{a}$ & $3,67 \mathrm{~b}$ & 4,14 \\
Termoneutra & $3,83 \mathrm{a}$ & $4,02 \mathrm{a}$ & $4,40 \mathrm{~b}$ & $4,54 \mathrm{~b}$ & $3,96 \mathrm{a}$ & 4,21 \\
Fria & $3,86 \mathrm{a}$ & $3,97 \mathrm{a}$ & $4,37 \mathrm{~b}$ & $4,56 \mathrm{~b}$ & $3,87 \mathrm{a}$ & 4,21 \\
\hline Fonte de Selênio & & & & & & \\
\hline Inorgânico & 3,73 & 3,92 & 4,44 & 4,61 & 3,82 & 4,18 \\
Orgânico & 3,80 & 3,93 & 4,43 & 4,58 & 3,84 & 4,19 \\
\hline Nivel de Selênio & \multicolumn{7}{c}{} & & & & \\
\hline Nivel "A." & 3,79 & 3,89 & 4,42 & 4,57 & 3,80 & 4,17 \\
Nivel "B" & 3,75 & 3,96 & 4,44 & 4,62 & 3,87 & 4,20 \\
\hline
\end{tabular}

Médias seguidas de letras distintas, dentro de cada fator, diferem entre si pelo teste de Tukey $(5 \%)$.

*Nível A: 0,15 mg de Se/kg de ração (1 - 21 dias de idade) e 0,20 mg (21- 42 dias de idade)

Nível B: 0,30 mg de Se/kg de ração (1 - 21 dias de idade) e 0,40 mg (21- 42 dias de idade) 
TABELA 4 - COMPRIMENTO DE PENA DO DORSO (REGIÃO DORSOPÉLVICA), DA PERNA (REGIÃO FEMORAL) E $2^{\circ}$ RÊMIGE PRIMARIA DE FRANGOS DE CORTE DE 35 E 43 DIAS DE IDADE, ALIMENTADOS COM DIETAS SUPLEMENTADAS COM DIFERENTES NÍVEIS DE SELÊNIO, NA FORMA ORGÂNICA OU INORGÂNICA E CRIADOS EM TEMPERATURAS DISTINTAS.

\begin{tabular}{lccc|ccc}
\hline & \multicolumn{6}{c}{ Comprimento de penas (mm) } \\
\hline & \multicolumn{7}{c}{35 dias } & \multicolumn{4}{c}{43 dias } \\
\hline Temperatura ambiente & Dorso & Perna & Rêmige & Dorso & Perna & Rêmige \\
\hline Fria & 38 a & 63 a & 124 & 45 a & 85 a & 149 \\
Termoneutra & 40 a & 66 a & 125 & 47 a & 84 a & 149 \\
Quente & $35 \mathrm{~b}$ & $56 \mathrm{~b}$ & 124 & $42 \mathrm{~b}$ & $80 \mathrm{~b}$ & 144 \\
\hline Nivel de Selênio & 40 & 64 & 125 & 44 & 80 & 147 \\
\hline Nivel "A" & 38 & 60 & 126 & 45 & 84 & 144 \\
Nível "B" & 39 & 58 & 124 & 46 & 84 & 150 \\
\hline Fonte de Selênio & 38 & 60 & 123 & 44 & 83 & 148 \\
\hline Inorgânico &
\end{tabular}

Médias seguidas de letras distintas, dentro de cada fator, diferem entre si pelo teste de Tukey (5\%). ${ }^{*}$ Nível A: 0,15 mg de Se/kg de ração (1 - 21 dias de idade) e 0,20 mg (21- 42 dias de idade) Nível B: 0,30 mg de Se/kg de ração (1 - 21 dias de idade) e 0,40 mg (21- 42 dias de idade)

\section{Conclusões}

A temperatura de criação é um componente de suma importância no desempenho de frangos, considerados como os mais importantes no controle dos processos metabólicos das aves, e principalmente no crescimento de penas, objeto de estudo deste ensaio.

A qualidade do empenamento é melhor otimizada pelo uso de Se orgânico como fonte de suplementação, em uma fase crítica de homeostase térmica do frango de corte, isto é na fase inicial, até 21 dias de vida pós-natal.

\section{REFERÊNCIAS}

BECKETT, G.J.; RUSSEL, A.; NICOL, F.; SAHU, P.; WOLF, C.R.; ARTHUR, A.R. Effect of selenium deficiency on hepatic type I 5'iodothyrinine deiodinase activity and hepatic tyroid hormone levels in rat. Biochemical Journal, Portland, v.282, p.483487, 1992.

COOPER, M.A.; WASHBURN, K.W. The relationships pf body temperature to weight gain, feed consumption and feed utilization in broiler under heat stress. Poultry Science, Champaign, v.77, p.237242, 1998.
EDENS, F.W.; CARTER, T.A.; PARKHURST, C.R. Effect of selenium source and litter type on broiler feathering. Journal of Applied Poultry Research, Athens, v.9, p.407-413, 2000.

EDENS, F.; PARHURST, C.R.; HAVENSTEIN, G.B. Housing and selenium influences on feathering in broilers. Journal of Applied Poultry Research, Athens, v.10, p.128-134, 2001.

GERAERT, P.A.; PADILHA, J.C.F.; GUILLAUMIN, $S$. Metabolic and endocrine changes induced by chronic heat exposure in broiler chickens: growth performance, body composition and energy retention. British Poultry Science, London, v.75, p.195-204,1996.

HADDAD E.E.; MASHALY M.M. Effect of thyroidectomy of immature male chickens on circulatig thyroid hormones and on response to thyroid-stimulating hormones and chronic cold exposure. Poultry Science, Champaign, v.68, p.169176, 1989.

HALPIN, K.M.; BAKER, D.H. Selenium deficiency and transsulfuration in the chick. The Journal of Nutrition, Mesa, v.114, p.606-612, 1984.

HAYASHI, K. Roles of thyroid hormone in growth and protein turnover. Animal Science and technology, Tokyo, v.64, p.938-947, 1993. 
Suplementação dietética de selênio para frangos de corte e seus efeitos sobre o empenamento...

JENSEN, L.S.; COLNAGO, G.L.; TAKAHASHI, K.; AKIBA, Y. Dietary selenium status and plasma thyroid hormones in chicks. Biological Trace Element Research, Totowa, v.10, p.11-18, 1986.

JIANHUA, H.; OHTSUKA, A.; HAYASHI, K. Selenium influences growth via thyroid hormone status in broiler chickens. British Poultry Science, London, v.84, p.727-732, 2000.

LAWRENCE T.L.J.; FOWLER V.R. Hormonal, genetic and immunological influences on growth. In: LAWRENCE, T.L.J.; FOWLER, V.R. Growth of farm animals. New York: Cab International,1997. p.102-149. LEESON, S.; SUMMERS, J.D. Commercial poultry nutrition. 2.ed. New York: State Manual Book \& Periodical Services, 1997. 324 p.

LUCAS, A.M.; STETTENHEIM, P.R. Avian anatomy integument. Michigan: Agriculture Handbook, 1972. $1320 \mathrm{p}$.

MENDES, A.A. Rendimento e qualidade de carcaça de frangos de corte. In.: CONFERÊNCIA APINCO DE CIÊNCIA E TECNOLOGIA AVÍCOLAS, 2001. Anais... Campinas: Facta, 2001. p.57-64.
ÖZKAN, S.; SMITH, W.K.; BATH, H.M. The development of terminal resistance of the feather coat in broilers with different feathering genotypes and feeding regimes. British Poultry Science, London, v.43, p.472-481, 2002.

PATON, N.D.; CANTOR, A.H.; PESCATORE, A.J.; FORD, M.J.; SMITH, C.A. The effect of dietary selenium source and level on the uptake of selenium by developing chick embryos. Poultry Science, Champaign, v.81, p.15481554, 2002.

SAS INSTITUTE (Cary, United States). SAS ${ }^{\circledR}$ User's Guide: Statistics. Cary, 2002.

WYLIE, L.M.; ROBERTSON, G.W.; MACLEOD, M.G.; HOCKING, P.M. Effects of ambient temperature and restricted feeding on growth of feathers in growing turkeys. British Poultry Science, London, v.42, p.449-455, 2001.

YALCIN, S.; SELTAR, P.; OZKAN, S.; CHANER, A. Comparative evaluation of three commercial broiler stocks in hot temperature climates. Poultry Science, Champaign, v.76, p.921-929, 1997.

Recebido para publicação: 25/01/2005

Aprovado:

$13 / 04 / 2005$ 